\title{
Lipid-lowering therapy and peripheral sensory neuropathy in type 2 diabetes: the Fremantle Diabetes Study
}

\author{
T. M. E. Davis • B. B. Yeap • W. A. Davis • D. G. Bruce
}

Received: 31 October 2007 / Accepted: 12 December 2007 /Published online: 11 January 2008

(C) Springer-Verlag 2007

\begin{abstract}
Aims/hypothesis The aim of this study was to assess the relationships between lipid-lowering therapy and the prevalence and incidence of peripheral sensory neuropathy in type 2 diabetes mellitus.

Methods We analysed data from an observational cohort study, the Fremantle Diabetes Study (FDS), specifically, (1) a cross-sectional sample comprising 1,237 FDS participants with type 2 diabetes mellitus, and (2) a longitudinal subgroup of 531 individuals who had attended six consecutive annual assessments. Neuropathy was identified using the clinical portion of the Michigan Neuropathy Screening Instrument.

Results At entry, the cross-sectional sample had a mean \pm SD age of $63.8 \pm 11.3$ years, $48.7 \%$ were men, median (interquartile range) diabetes duration was $4.0(1.0-9.0)$ years, and $30.9 \%$ had peripheral neuropathy. Fibrates and statins were used by 3.5 and $6.8 \%$, respectively. Multiple logistic regression analysis showed that older age, longer diabetes duration, central adiposity, increased height, higher fasting serum glucose, albuminuria and aboriginality were significant independent positive predictors of prevalent neuropathy, while systolic blood pressure and fibrate use (odds ratio $0.30,95 \%$ CI $0.10-0.86 ; p=0.025)$ were negatively associated. In the longitudinal subgroup, fibrate and statin use increased to 10.4 and $36.5 \%$, respectively, over 5 years. In
\end{abstract}

Electronic supplementary material The online version of this article (doi:10.1007/s00125-007-0919-2) contains supplementary material, which is available to authorised users.

T. M. E. Davis $(\bowtie) \cdot$ B. B. Yeap · W. A. Davis $\cdot$ D. G. Bruce School of Medicine and Pharmacology,

University of Western Australia,

Fremantle Hospital,

Fremantle, WA, Australia

e-mail: tdavis@cyllene.uwa.edu.au time-dependent Cox proportional hazards modelling, fibrate use [hazard ratio (HR) $0.52,95 \%$ CI $0.27-0.98$ ] and statin use (HR $0.65,95 \%$ CI $0.46-0.93$ ) were significant determinants of incident neuropathy $(p \leq 0.042)$.

Conclusions/interpretation These preliminary observational data suggest that therapy with a statin or a fibrate may protect against the development of diabetic peripheral sensory neuropathy, but there is a need for additional confirmatory evidence, preferably from randomised clinical trials.

Keywords Fibrate - Peripheral sensory neuropathy · Statin . Type 2 diabetes mellitus
Abbreviations
FDS Fremantle Diabetes Study
FIELD Fenofibrate and Event-Lowering in Diabetes
HR hazard ratio
MNSI Michigan Neuropathy Screening Instrument

\section{Introduction}

Epidemiological studies have suggested that dyslipidaemia is a risk factor for diabetic neuropathy [1], and case series dating from the 1960s [2] have provided evidence that lipid-lowering therapy might be neuroprotective. However, the use of fibrates and statins is also associated with an increased risk of reversible peripheral neuropathy in the general population [3]. Because of these apparently contradictory findings and the paucity of diabetes-specific descriptive data, we have assessed the relationship between serum lipids, lipid-lowering therapy and the prevalence and incidence of peripheral neuropathy in a representative, wellcharacterised cohort of patients with type 2 diabetes mellitus. 


\section{Methods}

Patients The Fremantle Diabetes Study (FDS) was a longitudinal observational study conducted in a community of 120,097 people. Details of recruitment and sample characteristics (including non-recruited subjects) have been published elsewhere [4]. Of 2,258 patients identified between 1993 and 1996, 1,426 (63\%) were recruited and 1,294 had type 2 diabetes [Electronic supplementary material (ESM) Table 1]. The FDS protocol was approved by the Fremantle Hospital Human Rights Committee and all participants gave informed consent. Annual follow-up continued until November 2001. The cohort of FDS patients with type 2 diabetes contained 531 patients with complete data at baseline and at least five subsequent consecutive annual reviews. Compared with the 763 remaining participants with type 2 diabetes, these patients were younger and more often male, and had shorter diabetes duration, lower $\mathrm{HbA}_{1 \mathrm{c}}$ levels, fewer vascular complications and lower subsequent mortality $(p \leq 0.001)$.

Baseline and annual assessments Patient assessment at baseline and each review included a questionnaire and physical examination [4]. Prescribed therapies were documented at each visit and missing details collected by telephone and/or review of hospital records. Lipid-lowering drugs available during all/part of the FDS were fluvastatin, simvastatin, pravastatin, cerivastatin, atorvastatin, gemfibrozil and fenofibrate. Biochemical tests were performed on fasting blood and urine using standard methods [4]. Serum cholesterol, HDL-cholesterol and triacylglycerol were measured enzymatically and the serum LDL-cholesterol calculated using the Friedewald equation for serum triacylglycerol $<4.5 \mathrm{mmol} / \mathrm{l}$.

\section{Definition of sensory neuropathy and other complications} Neuropathy was defined using the clinical portion of the Michigan Neuropathy Screening Instrument (MNSI), which has high diagnostic sensitivity and specificity for distal symmetrical polyneuropathy, including patients with diabetes [5]. It incorporates assessment of both feet for skin changes/ulceration, reflexes and vibration perception, generating a score out of 8 points (ESM Table 2). Scores $>2$ (or $>1$ for single-leg amputees) indicate neuropathy. Other complications were identified using standard procedures (ESM Table 3).

Statistical analysis SPSS statistical software (version 14.0, for Windows; SPSS, Chicago, IL, USA) was used for statistical analysis. Univariate and independent baseline associates of prevalent and incident neuropathy were determined using logistic regression analysis and Cox proportional hazards modelling, respectively. Because of increased use during follow-up, statin and fibrate therapy were considered as time-dependent covariates using a step function at the time of initiation. In both analyses, forward conditional modelling was employed.

\section{Results}

Cross-sectional data Data allowing characterisation of neuropathy were available for 1,237 participants with type 2 diabetes ( $95.6 \%$ of the total sample). At baseline, they were aged $($ mean \pm SD) $63.8 \pm 11.3$ years, $48.7 \%$ were men, their median diabetes duration was 4.0 years (interquartile range, 1.0-9.0 years) and $30.9 \%$ had peripheral neuropathy. Univariate associates of prevalent neuropathy are summarised in Table 1 . All potential baseline risk factors with $p<0.20$ in univariate logistic regression (ESM Table 4) were considered for entry into the multivariate analysis (Table 2), except that (1) the ratecorrected electrocardiographic QT interval was excluded because it was measured in only $60.0 \%$ of participants; and (2) aboriginality was included despite $p>0.20$ because this group was young and considered high-risk. Fibrate therapy was independently associated with less than one-third the risk of prevalent neuropathy.

Longitudinal data There were $136(25.6 \%)$ in the 5 year cohort with peripheral neuropathy at baseline. Of the remaining 395 patients, 248 (62.3\%) developed neuropathy subsequently (crude incidence 16.4\%/patient-year). As with prevalent neuropathy, univariate analysis revealed that those with incident neuropathy were older and had longer diabetes duration, worse blood pressure and glycaemic control, and a higher urinary albumin:creatinine at baseline (Table 1). There were additional socioeconomic/ethnic differences not observed in the cross-sectional analyses, but neither baseline fibrate nor statin use was associated with subsequent neuropathy.

During follow-up, the use of lipid-lowering therapy increased in the patients without neuropathy at baseline. There were $24(6.1 \%)$ on fibrates, $30(7.6 \%)$ on statin therapy and $1(0.3 \%)$ on combination statin/fibrate therapy at entry, increasing to $35(8.9 \%), 138(34.9 \%)$ and $6(1.5 \%)$, respectively, $(p<0.001)$ after 5 years. All baseline risk factors with $p<0.20$ in univariate regression (ESM Table 4) were considered for entry into the time-dependent Cox proportional hazards model of incident neuropathy (Table 3 ). Both time-dependent statin and fibrate use were negatively associated with new neuropathy, with hazard ratio (HR) reductions of nearly two-thirds and one-half, respectively. After further adjustment for time-dependent serum lipid concentrations, time-dependent use of statin $(p=0.018)$ but 
Table 1 Characteristics of all 1,237 FDS patients with valid neuropathy data at baseline and the 395 patients in the 5 year subgroup without neuropathy at baseline who did or did not develop this complication during follow-up

\begin{tabular}{|c|c|c|c|c|}
\hline & \multicolumn{2}{|l|}{ Baseline } & \multicolumn{2}{|l|}{5 year cohort } \\
\hline & No neuropathy & Neuropathy & No neuropathy & New neuropathy \\
\hline Number, $(\%)$ & $855(69.1)$ & $382(30.9)$ & $147(37.7)$ & $248(62.3)$ \\
\hline Age (years) & $61.5(11.2)$ & $69.0(9.6)^{* * *}$ & $56.9(9.4)$ & $63.6(8.7)^{* * *}$ \\
\hline Men $(\%)$ & 46.0 & $55.0 * *$ & 53.7 & 50.0 \\
\hline Diabetes duration (years), median (IQR) & $3.0(0.7-7.0)$ & $5.0(2.1-11.0)^{* * *}$ & $2.0(0.6-5.0)$ & $3.0(0.5-7.0)^{*}$ \\
\hline Height $(\mathrm{m})$ & $1.64(0.10)$ & $1.66(0.10)^{* *}$ & $1.65(0.08)$ & $1.66(0.10)$ \\
\hline $\operatorname{BMI}\left(\mathrm{kg} / \mathrm{m}^{2}\right)$ & $29.7(5.5)$ & $29.1(5.2)$ & $29.2(5.5)$ & $29.2(4.6)$ \\
\hline Waist circumference $(\% \text { overweight/obese })^{\mathrm{a}}$ & 85.2 & 88.2 & 78.8 & $87.9^{*}$ \\
\hline Fasting serum glucose $(\mathrm{mmol} / \mathrm{l})$, median (IQR) & $8.2(6.8-10.5)$ & $9.0(7.0-11.7)^{* * *}$ & $7.5(6.3-9.5)$ & $8.4(7.0-10.3)^{* *}$ \\
\hline $\mathrm{HbA}_{1 \mathrm{c}}(\%)$, median (IQR) & $7.3(6.3-8.7)$ & $7.8(6.6-9.2)^{* *}$ & $6.7(6.0-8.0)$ & $7.3(6.5-8.6)^{* *}$ \\
\hline \multicolumn{5}{|l|}{ Diabetes treatment $(\%)$} \\
\hline $\operatorname{Diet}^{\mathrm{b}}$ & 36.5 & 23.2 & 40.4 & 37.5 \\
\hline Oral hypoglycaemic agents & 54.9 & $60.2 * * *$ & 54.1 & 54.0 \\
\hline Insulin \pm oral hypoglycaemic agents & 8.6 & $16.6^{* * *}$ & 5.5 & 8.5 \\
\hline \multicolumn{5}{|l|}{ Blood pressure $(\mathrm{mmHg})$} \\
\hline Systolic & $149(23)$ & $153(24)^{* *}$ & $143(19)$ & $148(22)^{*}$ \\
\hline Diastolic & $81(11)$ & $80(12)$ & $81(10)$ & $80(10)$ \\
\hline On blood pressure-lowering medication (\%) & 47.6 & $57.3 * *$ & 40.8 & 46.4 \\
\hline Angiotensin converting enzyme inhibitor $(\%)$ & 19.8 & $25.9^{*}$ & 17.0 & 19.4 \\
\hline Total serum cholesterol $(\mathrm{mmol} / \mathrm{l})$ & $5.5(1.1)$ & $5.5(1.1)$ & $5.5(1.2)$ & $5.4(0.9)$ \\
\hline Serum HDL-cholesterol (mmol/l) & $1.05(0.33)$ & $1.06(0.32)$ & $1.08(0.34)$ & $1.05(0.32)$ \\
\hline Serum LDL-cholesterol $(\mathrm{mmol} / \mathrm{l})^{\mathrm{c}}$ & $3.5(0.9)$ & $3.5(0.9)$ & $3.4(0.9)$ & $3.4(0.8)$ \\
\hline Serum triacylglycerol $(\mathrm{mmol} / \mathrm{l})$ & $1.9(1.1-3.4)$ & $1.9(1.1-3.2)$ & $2.0(1.1-3.5)$ & $1.8(1.0-3.1)$ \\
\hline \multicolumn{5}{|l|}{ On lipid-lowering medication (\%) } \\
\hline Any & 12.3 & $6.6^{* *}$ & 16.3 & 12.9 \\
\hline Statins & 7.5 & 5.2 & 9.5 & 6.9 \\
\hline Fibrates & 4.6 & $1.0 * *$ & 7.5 & 5.6 \\
\hline Urinary albumin:creatinine (mg/mmol) & $2.5(0.6-9.9)$ & $4.6(1.0-21.7)^{* * *}$ & $1.6(0.6-4.7)$ & $2.3(0.7-7.9)^{* *}$ \\
\hline Retinopathy (\%) & 12.3 & $22.8^{* * *}$ & 6.8 & 12.2 \\
\hline Coronary heart disease $(\%)$ & 29.2 & $36.1^{*}$ & 20.7 & $30.9 *$ \\
\hline Cerebrovascular disease $(\%)$ & 9.1 & 11.0 & 2.7 & 6.9 \\
\hline Peripheral arterial disease (\%) & 25.9 & $35.2 * *$ & 16.3 & 24.9 \\
\hline Rate-corrected QT interval $\left(\mathrm{m} / \mathrm{s}^{0.5}\right)^{\mathrm{d}}$ & $410(27)$ & $419(32)^{* * *}$ & $403(24)$ & $405(25)$ \\
\hline \multicolumn{5}{|l|}{ Ethnic background } \\
\hline Anglo-Celt ${ }^{\mathrm{b}}$ & 61.1 & 67.0 & 70.7 & 64.5 \\
\hline Southern European & 19.9 & $15.2^{*}$ & 14.3 & 21.8 \\
\hline Other European & 8.5 & 8.4 & 6.1 & 9.7 \\
\hline Asian & 3.5 & 3.1 & 5.4 & $0.8^{*}$ \\
\hline Mixed/other & 5.8 & 4.5 & 3.4 & 2.8 \\
\hline Aboriginal & 1.2 & 1.8 & 0 & 0.4 \\
\hline Limited English-speaking ability (\%) & 15.3 & 15.5 & 9.5 & 14.9 \\
\hline Educated beyond primary school level (\%) & 75.5 & 71.0 & 84.2 & $73.8^{*}$ \\
\hline Married/de facto relationship (\%) & 67.2 & 64.2 & 80.3 & $69.0^{*}$ \\
\hline Any exercise in past 2 weeks (\%) & 75.0 & $66.0 * * *$ & 84.9 & 78.9 \\
\hline \multicolumn{5}{|l|}{ Smoking status $(\%)$} \\
\hline Never $^{\mathrm{b}}$ & 46.6 & 40.7 & 45.6 & 49.8 \\
\hline Ex & 37.5 & $46.0^{*}$ & 42.9 & 36.8 \\
\hline Current & 15.9 & 13.3 & 11.6 & 13.4 \\
\hline Alcohol consumption (standard drinks/day), median (IQR) & $0(0-0.8)$ & $0(0-0.8)$ & $0(0-0.8)$ & $0(0-0.8)$ \\
\hline
\end{tabular}

Data are means (SD) or geometric mean (SD range) unless otherwise stated

${ }^{\mathrm{a}}$ Waist circumference $\geq 94.0 \mathrm{~cm}$ (men), $\geq 80.0 \mathrm{~cm}$ (women)

${ }^{\mathrm{b}}$ Reference group

${ }^{\mathrm{c}} n=794(92.9 \%)$ without and $357(93.5 \%)$ with neuropathy at baseline, and $139(94.6 \%)$ and $231(93.1 \%)$, respectively, in the 5 year cohort

${ }^{\mathrm{d}} \mathrm{QT} / \sqrt{\mathrm{RR}}, n=499(58.4 \%)$ without and $243(63.6 \%)$ with neuropathy at baseline, and $74(50.3 \%)$ and $131(52.8 \%)$, respectively, in the 5 year cohort ${ }^{*} p<0.05,{ }^{* *} p<0.01,{ }^{* * *} p<0.001$ vs no neuropathy

$\mathrm{IQR}$, interquartile range 
Table 2 Independent risk factors for prevalent neuropathy as identified by multiple logistic regression analysis in 1,237 patients with type 2 diabetes

${ }^{a}$ A 2.72-fold increase in urinary albumin:creatinine ratio corresponds to an increase of 1.0 in $\log _{\mathrm{e}}$ (albumin:creatinine ratio)

\begin{tabular}{lll}
\hline Prevalent neuropathy & Odds ratio $(95 \% \mathrm{CI})$ & $p$ value \\
\hline Age (increase of 10 years) & $2.28(1.93-2.69)$ & $<0.001$ \\
Height (increase of $1 \mathrm{~cm})$ & $1.04(1.03-1.06)$ & $<0.001$ \\
Diabetes duration (increase of 5 years) $_{\text {Log }_{\mathrm{e}}(\text { urinary albumin:creatinine) }}^{\mathrm{a}}(\mathrm{mg} / \mathrm{mmol})$ & $1.18(1.07-1.31)$ & 0.001 \\
Fasting serum glucose (increase of $1 \mathrm{mmol} / \mathrm{l})$ & $1.18(1.07-1.30)$ & 0.001 \\
Systolic blood pressure (increase of $10 \mathrm{mmHg})$ & $1.08(1.03-1.12)$ & 0.001 \\
On fibrate therapy & $0.93(0.87-0.99)$ & 0.020 \\
Aboriginal background & $0.30(0.10-0.86)$ & 0.025 \\
Overweight/obese (by waist circumference) & $3.70(1.17-11.70)$ & 0.026 \\
& $1.58(1.05-2.39)$ & 0.029
\end{tabular}

not fibrate $(p=0.09)$ therapy remained negatively associated with neuropathy (ESM Table 5).

\section{Discussion}

The present study provides preliminary observational evidence that lipid-lowering therapy may protect against peripheral sensory neuropathy complicating type 2 diabetes. This apparent benefit was observed in both crosssectional and longitudinal analyses, was independent of other determinants of neuropathy, and is consistent with evidence from in vitro and animal studies that lipidlowering therapy has multiple neuroprotective effects through improvement in Schwann cell [6] and polyol pathway [7] function, and improved neuronal blood supply [6]. Our findings should also help allay fears, based largely on case reports [3], that these drugs could increase the incidence of neuropathy and associated complications such as ulceration and amputation in diabetic patients.

In the placebo-controlled Fenofibrate and Event-Lowering in Diabetes (FIELD) study of fenofibrate for cardiovascular disease in type 2 diabetes [8], laser-treated retinopathy and microalbuminuria were significantly reduced in those allocated active drug. In the present study and the FIELD study, the lack of a specific indication for

Table 3 Independent risk factors for incident neuropathy in 395 patients initially free of neuropathy as identified by Cox proportional hazards modelling including time-dependent fibrate and statin use

\begin{tabular}{lll}
\hline Incident neuropathy & HR $(95 \% \mathrm{CI})$ & $p$ value \\
\hline Age (increase of 10 years) & $1.86(1.60-2.15)$ & $<0.001$ \\
Other European ethnicity & $1.77(1.15-2.72)$ & 0.010 \\
Asian ethnicity & $0.20(0.05-0.84)$ & 0.028 \\
Retinopathy (any) & $1.61(1.10-2.37)$ & 0.015 \\
Married/de facto relationship & $0.75(0.57-0.98)$ & 0.038 \\
Time-dependent fibrate use & $0.51(0.27-0.97)$ & 0.040 \\
Time-dependent statin use & $0.70(0.49-0.997)$ & 0.048 \\
\hline
\end{tabular}

statins or fibrates to be used for diabetic microangiopathy strengthens the case for an association between lipidlowering therapy and the prevention of these complications. It avoids potential allocation bias that would require careful case-control matching such as is used in propensity analysis of longitudinal observational data. Although neuropathy data have not been reported from the FIELD study [8] or major statin treatment trials, the detailed assessments required for instruments such as MNSI are unlikely to have been performed.

In the Steno 2 study in type 2 diabetes [9], intensive multifactorial therapy included a significantly greater use of statins but not fibrates compared with conventional management. Intensive therapy reduced the risk of autonomic neuropathy but not peripheral neuropathy. This result is at odds with the present data and, since the intensive group had a mean $0.7 \%$ lower $\mathrm{HbA}_{1 \mathrm{c}}$ at the end of the trial, with epidemiological data showing a strong link between glycaemia and peripheral neuropathy [1]. Steno 2 had a relatively small sample size ( 80 per group) and the assessment of neuropathy was limited to biothesiometry, factors that may help to explain these apparent discrepancies.

The present cross-sectional and longitudinal data (although the latter were derived from surviving patients) confirm that peripheral sensory neuropathy is common, afflicting $>50 \%$ of type 2 patients 8 years after diagnosis. We found evidence that particular ethnic/racial groups were at risk, including Australian aborigines. Our data also suggest that overweight/obesity may be an additional modifiable risk factor for diabetic neuropathy. The apparently protective effect of systolic hypertension in crosssectional analysis is difficult to explain but there was no such association with incident neuropathy. We did not find an independent association between baseline serum lipid parameters and neuropathy in our patients, but previously published studies showing such a relationship were done at a time when the use of lipid-lowering therapy was limited [1]. In addition, the present data are consistent with beneficial effects of hypolipidaemic treatment that are largely independent of effects on lipoprotein metabolism, and with statins and fibrates exhibiting separate protective mechanisms. 
The present study had limitations. FDS data are observational but, in the absence of data from randomised controlled trials, well-conducted observational studies can provide reasonable estimates of intervention effects [10]. In addition, the widespread evidence-based use of statins for prevention of cardiovascular disease in type 2 diabetes questions the ethics and feasibility of a randomised controlled trial with neuropathy as the primary endpoint. Reduced compliance with lipid-lowering therapy, especially in diabetic patients on a range of medications, would have led to an underestimate of possible neuroprotective effects of lipid-lowering therapy in the present study. It is also possible that some patients stopped lipid-lowering therapy because of neuropathic symptoms. However, the low rate of discontinuation from trials of lipid-lowering therapy was for reasons other than neuropathy [8]; only one patient in our 5 year subgroup was hospitalised because of symptomatic neuropathy during follow-up and this person was not taking lipid-lowering therapy at the time.

Our data are in accord with clinical observations [2], emerging preclinical data $[6,7]$ and indirect evidence relating to other microvascular complications [8] that lipid-lowering therapy may have benefits beyond its anti-atherogenic effects in type 2 diabetes. Confirmatory evidence of the neuroprotective effects of statins and fibrates may come from further in vitro and animal studies, and perhaps from clinical studies involving single and dual lipid-lowering therapy arms.

Acknowledgements We are grateful to the patients for their participation, and to the FDS staff for help with collecting and recording clinical information. We thank the Biochemistry Department at Fremantle Hospital and Health Service for performing laboratory tests, and the Diabetic Education, Podiatry and Dietetic Departments for assistance with recruitment of patients. The Fremantle Diabetes Study was supported by the Raine Foundation, University of Western Australia.

Duality of interest T. M. E. Davis has the following conflicts of interest that relate to manufacturers of lipid-lowering therapies used in type 2 diabetes: (1) Advisory Board membership - Pfizer; (2) sponsorship to attend national or international meetings - Pfizer, Merck, Solvay-Fournier; (3) funding for investigator-initiated research - Merck, Solvay-Fournier. B. B. Yeap has been an Advisory Board member for Astra-Zeneca. D. G. Bruce and W. A. Davis have no competing interests.

\section{References}

1. Girach A, Manner D, Porta M (2006) Diabetic microvascular complications: can patients at risk be identified? A review. Int J Clin Pract 60:1471-1483

2. Duncan GG, Elliott FA, Duncan TG, Schatanoff J (1968) Some clinical potentials of chlorophenoxyisobutyrate (Clofibrate) therapy. (Hyperlipidemia - angina pectoris - blood sludging diabetic neuropathy). Trans Am Clin Climatol Assoc 79:216-228

3. Corrao G, Zambon A, Bertu L, Botteri E, Leoni O, Contiero P (2004) Lipid lowering drugs prescription and the risk of peripheral neuropathy: an exploratory case-control study using automated databases. J Epidemiol Community Health 58:1047-1051

4. Davis TME, Zimmet P, Davis WA, Bruce DG, Fida S, Mackay IR (2000) Autoantibodies to glutamic acid decarboxylase in diabetic patients from a multiethnic Australian community: the Fremantle Diabetes Study. Diabet Med 17:667-674

5. Moghtaderi A, Bakhshipour A, Rashidi H (2006) Validation of Michigan neuropathy screening instrument for diabetic peripheral neuropathy. Clin Neurol Neurosurg 108:477-481

6. Ii M, Nishimura H, Kusano KF et al (2005) Neuronal nitric oxide synthase mediates statin-induced restoration of vasa nervorum and reversal of diabetic neuropathy. Circulation 112:93-102

7. Balendiran GK, Rajkumar B (2005) Fibrates inhibit aldose reductase activity in the forward and reverse reactions. Biochem Pharmacol 70:1653-1663

8. Keech A, Simes RJ, Barter P et al (2005) Effects of long-term fenofibrate therapy on cardiovascular events in 9795 people with type 2 diabetes mellitus (the FIELD study): randomised controlled trial. Lancet 366:1849-1861

9. Gaede P, Vedel P, Larsen N, Jensen GV, Parving HH, Pedersen O (2003) Multifactorial intervention and cardiovascular disease in patients with type 2 diabetes. N Engl J Med 348:383-393

10. Benson K, Hartz AJ (2000) A comparison of observational studies and randomized, controlled trials. N Engl J Med 342:1878-1886 